\title{
Grape-Seed Polyphenolic Extract Improves the Eye Phenotype in a Drosophila Model of Tauopathy
}

\author{
Cathie M. Pfleger, ${ }^{1}$ Jun Wang, ${ }^{2}$ Lauren Friedman, ${ }^{2}$ Roselle Vittorino, ${ }^{2}$ Lindsay M. Conley, ${ }^{2}$ \\ Lap Ho, ${ }^{2}$ Hayley C. Fivecoat, ${ }^{2}$ and Giulio M. Pasinetti ${ }^{2,3}$
}

${ }^{1}$ Department of Oncological Sciences, Mount Sinai School of Medicine, One Gustave L. Levy Place, NY 10029, USA
${ }^{2}$ Department of Neurology, The Mount Sinai School of Medicine, One Gustave L. Levy Place, NY 10029, USA
${ }^{3}$ James J. Peters Veteran Affairs Medical Center, 130 West Kingsbridge Road, Bronx, NY 10468, USA

Correspondence should be addressed to Giulio M. Pasinetti, giulio.pasinetti@mssm.edu

Received 8 April 2010; Revised 24 June 2010; Accepted 9 July 2010

Academic Editor: Gemma Casadesus

Copyright (c) 2010 Cathie M. Pfleger et al. This is an open access article distributed under the Creative Commons Attribution License, which permits unrestricted use, distribution, and reproduction in any medium, provided the original work is properly cited.

Drosophila models of tauopathies have been developed by transgenically overexpressing the disease-associated forms of tau. In this paper we report for the first time that a recently developed Grape-Seed Polyphenolic Extract (GSPE) improves the eye phenotype of a Drosophila eye model of $R 406 \mathrm{~W}$ tau. GSPE-mediated improvements in this distinct in vivo neurodegeneration model for protein misfolding/aggregation suggest that GSPE may have therapeutic value in disorders involving aberrant protein aggregation.

\section{Introduction}

Abnormal protein conformations resulting in misfolded and/or abnormally aggregated protein species are a characteristic feature of several neurodegenerative disorders. For example, the microtubule-associated protein tau adopts abnormal conformations resulting in protein helical filament/immunopositive neurofibrillary tangles (NFTs) and is a characteristic neuropathological feature in Alzheimer's Disease (AD), Pick's disease, progressive supranuclear palsy, and corticobasal ganglionic degeneration (CBD). Tau mutations cause frontotemporal dementia with Parkinsonism- (FTDP-) 17, proving that tau dysfunction can directly promote neurodegeneration [1].

Drosophila models using the transgenic Gal4/UAS system [2] to overexpress disease-associated aggregation-prone proteins have modeled aspects of tauopathy by overexpressing $R 406 \mathrm{~W}$ mutant tau $[3,4]$ and Huntington's disease (HD) by overexpressing Q93httexon1 [5-7] (for review, see [8]). Overexpressing $R 406 \mathrm{~W}$ in cells that form the eye (ey > $R 406 \mathrm{~W}$ ) leads to dramatic reduction in or complete absence of the eye. Eyes that do form demonstrate abnormal morphology [4]; for review, see [8].

We previously reported that moderate consumption of the red wine, Cabernet Sauvignon (Vitis vinifera), prevented abnormal $\beta$-amyloid $(\mathrm{A} \beta)$ oligomerization coincidental with a significant attenuation of spatial memory impairment in a mouse model of AD-type amyloid neuropathology [9]. Most importantly, we identified a polyphenolic compound highly concentrated in grape-seed polyphenolic extract (GSPE) as potentially responsible for the beneficial role of moderate consumption of red wine (Vitis vinifera) $[10,11]$.

More recently, Ho et al. reported that, using an in vitro aggregation assay, GSPE can significantly inhibit tau peptide Ac(306)VQIVYK(311) aggregation. Moreover, GSPE can also disaggregate preexisting aggregated tau peptides [12]. These results strongly suggest that GSPE might provide beneficial disease-modifying activity in tau-associated neurodegenerative disorders by modulating tau-mediated neuropathologic mechanisms. In this paper, we use the eye phenotype of a Drosophila model of mutant R406W tau to further evaluate the beneficial role of GSPE in tau-mediated neuropathology in vivo. We report for the first time that treatment with GSPE significantly benefits Drosophila phenotypes carrying mutant tau $(R 406 \mathrm{~W})$, further supporting the potential use in vivo of GSPE in abnormal tau aggregation, as previously demonstrated in vitro [12]. 


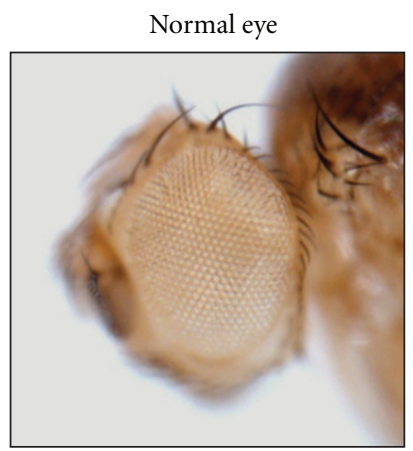

(a)

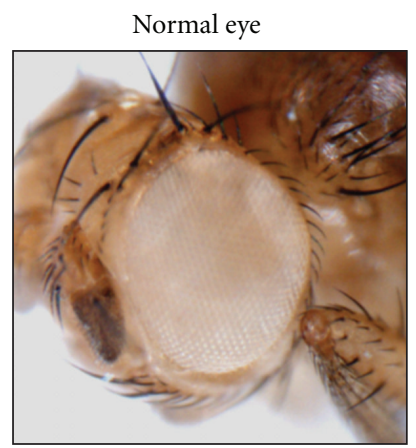

(d)

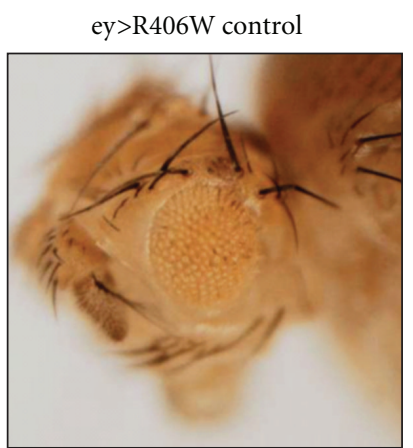

(b)

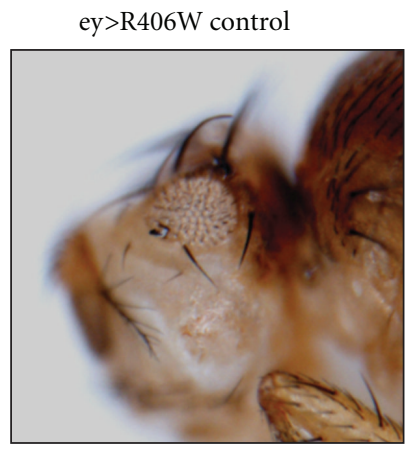

(e)

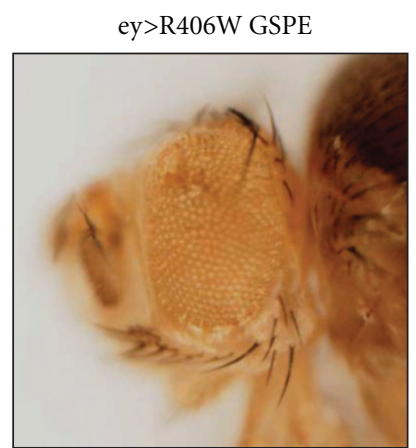

(c)

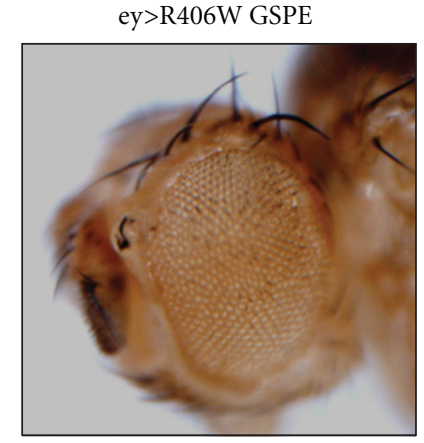

(f)

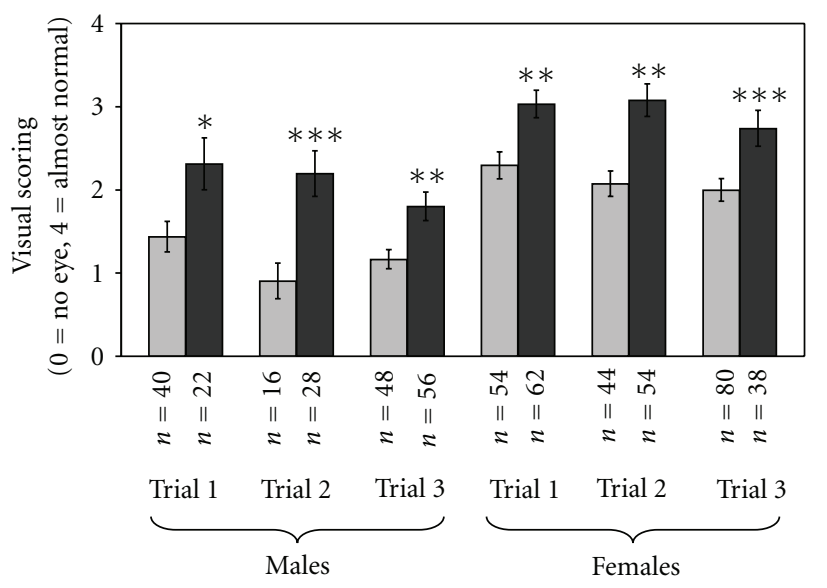

$\square$ Control

- GSPE-treated

(g)

Figure 1: GSPE attenuates R406W tau overexpression in the fly eye. Male and female eye size differs; male eyes are shown in (a)-(c), and female eyes are shown in (d)-(f). Overexpression of $R 406 \mathrm{~W}$ early in eye development results in a small or no eye ((b), (e)). Eyes that do not overexpress $R 406 \mathrm{~W}$ are shown in (a), (d) for comparison. GSPE treatment ameliorates the reduction in eye size (representative eyes shown in (c), (f)). GSPE treatment does not affect normal-eye development (not shown). (g) The range of ey $>R 406 \mathrm{~W}$ phenotypes varies between trials, so treatment comparisons were made within experiments. The average visual score $(0=$ no eye, $4=$ almost normal eye $) \pm$ SEM of male and female eyes is shown for three independent trials for males and three independent trials for females. The number of flies $(n)$ and $P$ value calculated using a paired $t$-test on GraphPad online software are indicated beneath each trial. Flies were collected within 5 days of eclosion. Statistically significant improvement $(P<.05)$ was observed in multiple independent experiments. ${ }^{*}$ indicates $P<.05, * *$ indicates $P<.01$, and ${ }^{* * *}$ indicates $P<.001$. (An excerpt of these images has been presented without data in a review manuscript by the author.) 


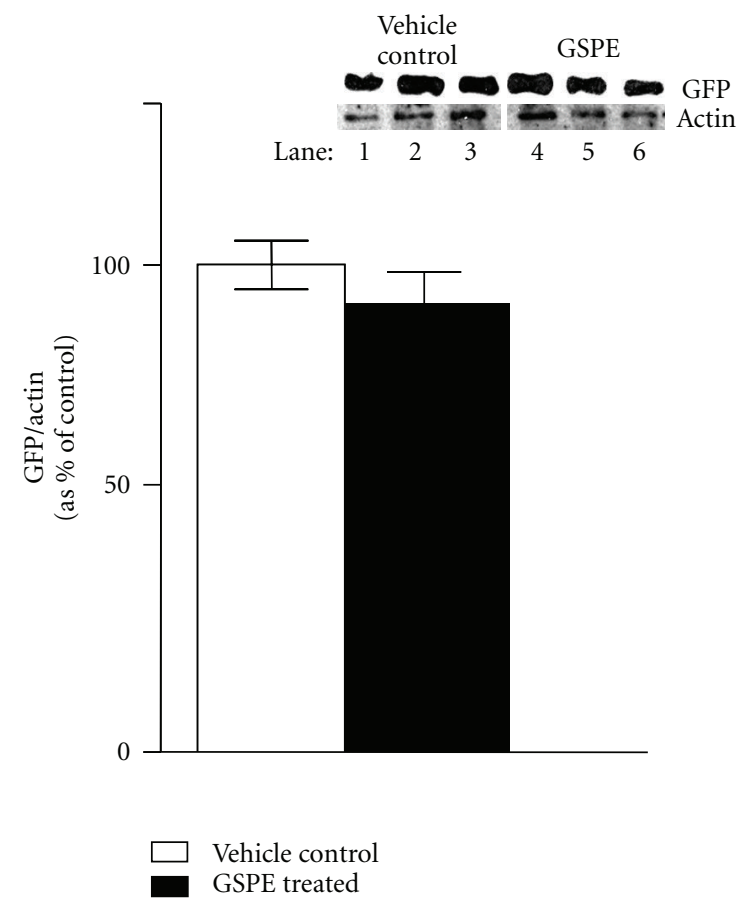

(a)

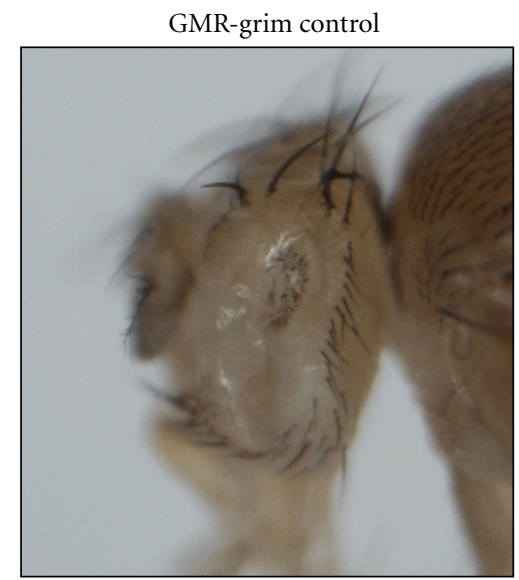

(b)

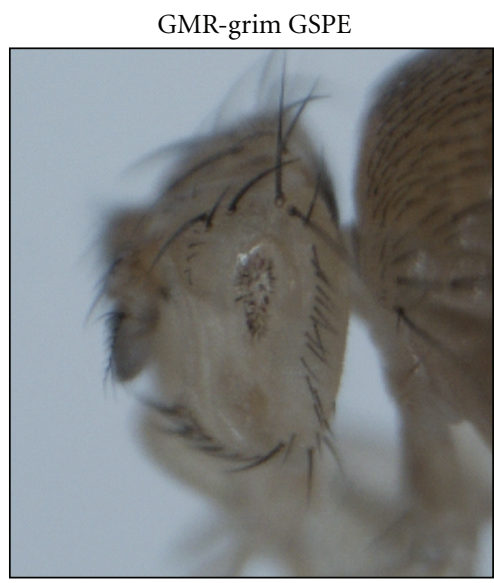

(c)

FIGURE 2: GSPE does not inhibit Gal4-mediated expression of GFP. (a) Western-blot analysis of lysates from TubGal4 > GFP flies (created by crossing TubGal4 flies to UAS-GFP flies) reared from egg deposition either on food supplemented with water (vehicle control) (Lanes 1-3) or reared on GSPE food (lanes 4-6). Each lysate was prepared from 6 fly heads, and three independent groups of 6 flies per group (lane) are shown. The same immunoblot was probed first with antibodies raised against GFP (upper panel) and then immunoblots were stripped and reprobed with antiactin antibodies (lower panel). (b) GMR-grim flies have very small, almost absent eyes when reared on control food. (c) Eye size is not affected when GMR-grim flies are reared on GSPE food.

\section{Materials and Methods}

In this study, MegaNatural grape-seed polyphenolic extract (GSPE) was provided by Polyphenolics, Inc. (Madera, CA), as highly purified ( $>97 \%$ total polyphenols) water-soluble polyphenolic preparation from Vitis vinifera seeds.

2.1. Drosophila Strains. w; eygal4/SM6-TM6B and GMRgrim flies were obtained from the lab of IK Hariharan; $w$; UAS
R406W and $w$; Tubgal4 (FlyBase ID = FBti0012687) and UAS green fluorescent protein (GFP) (FlyBase ID FBti0012686) elements were obtained from the Bloomington Stock Center.

2.2. R406W Tau Experiments and Visual Scoring of Eye Abnormality. eygal4/SM6-TM6B flies were crossed to UAS R406W flies to generate ey $>$ R406W flies. ey $>R 406 W$ eggs were laid in and reared on instant fly medium formula 424 supplemented with $2.8 \mu \mathrm{g} / \mathrm{mL}$ GSPE ("GSPE food"), the 
concentration used in our previous study [13], or control food supplemented with an equivalent volume of water (GSPE solvent, vehicle control).

Flies of the indicated genotypes were examined side by side under a dissecting scope. Eye regions which formed no ommatidia (therefore lacked eye tissue completely) were considered " $0=$ no eye" and required no comparison. For eye areas which did form obvious ommatidia/eye tissue, wild-type control flies were examined at the same time to establish the " $4=$ almost wild-type" eye size, shape, and pattern upper limit. Eyes that formed but did not reach the " 4 = almost wild-type" category were lined up under the scope by size and grouped into categories based on the amount of eye tissue present. Because eyes were grouped side by side under the microscope, relative sizes were easy to establish. When additional conditions or repeated experiments were examined, representative eyes of each category from the previous experiments were examined again in parallel to ensure that the same designations/scoring were maintained.

2.3. Statistical Analyses. The distribution of eye sizes was reviewed and found to have negative kurtosis, indicating a distribution that was more uniform over the range from 0 to 4 than a normal distribution. Negative kurtosis does not substantially impair analysis of variance.

In addition to overall analysis of variance (with trials nested within gender and crossed by treatment), separate $t$ tests were performed for each trial tested.

\section{Results}

3.1. GSPE Treatment Improves the Eye Phenotype of ey > R406W Flies. ey $>$ R406W flies show a range of phenotypes from no eye to small, abnormal eyes. The reduced size and abnormal morphology of ey $>R 406 \mathrm{~W}$ eyes were improved by $\operatorname{GSPE}(\mathrm{F}(1,531)=57.29 ; P<.0005)$. The incidence of the worst outcomes (visual score of 0 or 1) decreased upon GSPE treatment in each trial while the incidence of the best outcomes (visual score of 3 or 4 ) increased upon GSPE treatment in each trial. There were also differences in the size of male and female eyes $(\mathrm{F}(1,531)=58.62$; $P<.0005)$. Representative eyes are shown in Figures 1(a)1(f) ((a)-(c) male; (d)-(f) female). Nonetheless, there was no interaction between gender and treatment $(\mathrm{F}(1,531)$ $=0.30 ; P=.59)$; the difference between treated and untreated flies was similar in male and female flies. Separate $t$-tests showed that each of the six trials had a statistical difference with GSPE treatment characterized by increased visual scoring relative to untreated controls (Figure $1(\mathrm{~g})$ ). However, within the combinations of gender and treatment, the trials did not differ significantly $(\mathrm{F}(4,531)=1.90$; $P<.11)$.

\subsection{GSPE Effects on Gal4/UAS-Mediated Protein Expression.} To address the concern that GSPE could disrupt the production of the toxic proteins by the Gal4/UAS system, we performed control experiments using the Gal4/UAS system to determine whether GSPE treatment prevents expression of GFP using a UAS-GFP transgene and TubGal4 (constitutively expressed as Gal4). We found no detectable alteration in the production of GFP both by fluorescent imaging of GFP (not shown) and by Western analysis (Figure 2(a)).

3.3. GSPE Does Not Affect the Small-Eye Phenotype Caused by Overexpression of the Proapoptotic Gene Grim. To investigate if GSPE acts on cell death pathways directly, we examined the effects of GSPE treatment on flies overexpressing the proapoptotic gene grim in differentiating cells in the eye (which causes a very small eye). GMR-grim flies (which overexpress the pro-apoptotic gene grim in differentiating cells in the eye) laid eggs on control food or GSPE food. GMR-grim flies reared entirely on GSPE food showed no effect of reduced eye size (Figures 2(b)-2(c)).

\section{Discussion}

We have demonstrated that GSPE treatment benefits a distinct Drosophila model of neurodegeneration involving aggregation in vivo (Figures 1 and 2). GSPE showed no effect on Gal4-mediated expression of GFP (Figure 2(a)), indicating that GSPE does not block Gal4/UAS-dependent gene expression. GSPE had no effect on eye size in GMRgrim flies (which overexpress in the eye the pro-apoptotic gene grim which activates caspases and severely reduces eye size [14]) (Figures 2(b)-2(c)). Thus, although the mechanism remains unknown, GSPE-mediated improvements in the Drosophila eye model likely occur subsequent to production of the toxic protein but upstream of caspase activation.

Presently there is no treatment for the devastating progressive neurodegenerative disorders involving tau abnormalities. Continuing investigations suggest that oligomeric forms of these misfolded proteins may play a seminal role in disease etiology. Results from our studies show that GSPE may rescue abnormal tau phenotype in a Drosophila model of tauopathy. This study is consistent with our previous study, which provided evidence that GSPE could inhibit tau peptide aggregation [12] and prevent oligomerization of $\mathrm{A} \beta$ peptides into high molecular weight species in models of AD-amyloid neuropathology $[10,11]$. The study supports further investigations of GSPE in models of tauopathies, for example, frontotemporal dementia and CBD, among others.

\section{Acknowledgments}

This paper was generously supported in part by NIH P01 80004511 and Polyphenolics, Inc. (Madera, CA), to G.M. Pasinetti. Drs. Pasinetti, Wang, and Ho are named inventors of a pending patent application filed by Mount Sinai School of Medicine (MSSM) for grape-seed polyphenolic extract. In the event that the pending or issued patent is licensed, Drs. Pasinetti, Wang, and Ho would be entitled to a share of any proceeds MSSM receives from the license. 


\section{References}

[1] M. Hutton, "Missense and splicing mutations in Tau associated with FTDP-17: multiple pathogenic mechanisms," NeuroScience News, vol. 2, no. 3-4, pp. 73-82, 1999.

[2] A. H. Brand and N. Perrimon, "Targeted gene expression as a means of altering cell fates and generating dominant phenotypes," Development, vol. 118, no. 2, pp. 401-415, 1993.

[3] C. W. Wittmann, M. F. Wszolek, J. M. Shulman et al., "Tauopathy in Drosophila: neurodegeneration without neurofibrillary tangles," Science, vol. 293, no. 5530, pp. 711-714, 2001.

[4] Z. Berger, B. Ravikumar, F. M. Menzies et al., "Rapamycin alleviates toxicity of different aggregate-prone proteins," Human Molecular Genetics, vol. 15, no. 3, pp. 433-442, 2006.

[5] N. Agrawal, J. Pallos, N. Slepko et al., "Identification of combinatorial drug regimens for treatment of Huntington's disease using Drosophila," Proceedings of the National Academy of Sciences of the United States of America, vol. 102, no. 10, pp. 3777-3781, 2005.

[6] J. S. Steffan, A. Kazantsev, O. Spasic-Boskovic et al., "The Huntington's disease protein interacts with p53 and CREBbinding protein and represses transcription," Proceedings of the National Academy of Sciences of the United States of America, vol. 97, no. 12, pp. 6763-6768, 2000.

[7] W. J. Wolfgang, T. W. Miller, J. M. Webster et al., "Suppression of Huntington's disease pathology in Drosophila by human single-chain Fv antibodies," Proceedings of the National Academy of Sciences of the United States of America, vol. 102, no. 32, pp. 11563-11568, 2005.

[8] T.-K. Sang and G. R. Jackson, "Drosophila models of neurodegenerative disease," NeuroRx, vol. 2, no. 3, pp. 438-446, 2005.

[9] J. Wang, L. Ho, Z. Zhao et al., "Moderate consumption of Cabernet Sauvignon attenuates $\mathrm{A} \beta$ neuropathology in a mouse model of Alzheimer's disease," The FASEB Journal, vol. 20, no. 13, pp. 2313-2320, 2006.

[10] K. Ono, M. M. Condron, L. Ho et al., "Effects of grape seedderived polyphenols on amyloid $\beta$-protein self-assembly and cytotoxicity," The Journal of Biological Chemistry, vol. 283, no. 47, pp. 32176-32187, 2008.

[11] J. Wang, L. Ho, W. Zhao et al., "Grape-derived polyphenolics prevent $\mathrm{A} \beta$ oligomerization and attenuate cognitive deterioration in a mouse model of Alzheimer's disease," Journal of Neuroscience, vol. 28, no. 25, pp. 6388-6392, 2008.

[12] L. Ho, S. Yemul, J. Wang, et al., "Grape seed polyphenolic extract (GSE) as a novel therapeutic reagent in tau-mediated neurodegenerative disorders," Journal of Alzheimer's Disease, vol. 16, pp. 433-439, 2009.

[13] J. Wang, C. Pfleger, L. Friedman, et al., "Potential application of grape derived polyphenols in Huntington's disease," Translational Neuroscience. In press.

[14] P. Chen, W. Nordstrom, B. Gish, and J. M. Abrams, "grim, a novel cell death gene in Drosophila," Genes and Development, vol. 10, no. 14, pp. 1773-1782, 1996. 


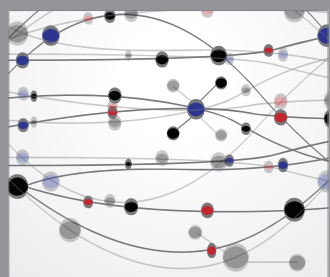

The Scientific World Journal
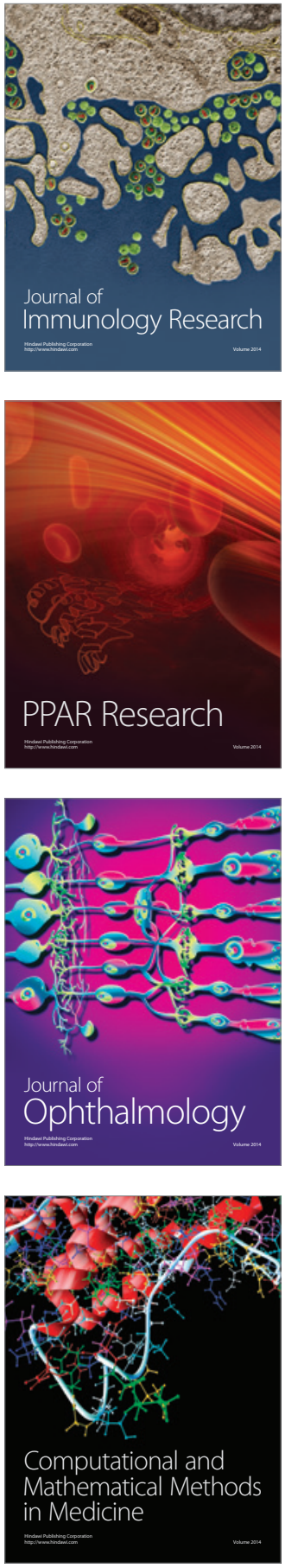

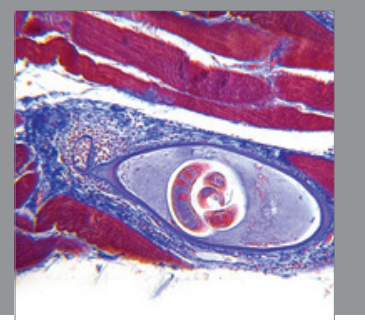

Gastroenterology

Research and Practice
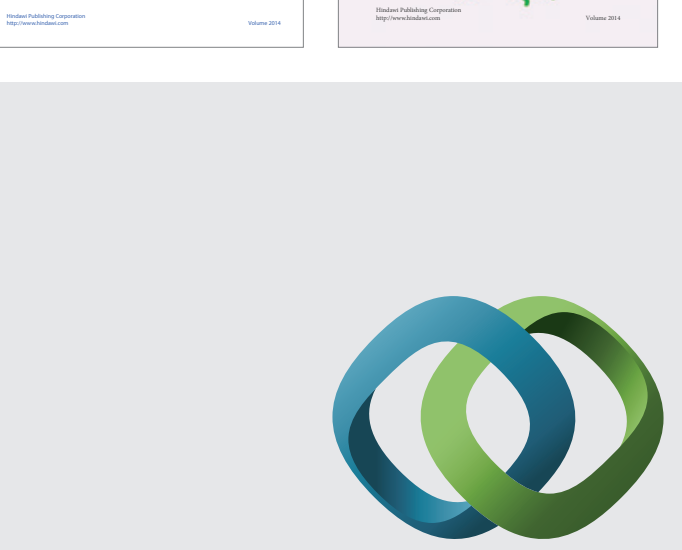

\section{Hindawi}

Submit your manuscripts at

http://www.hindawi.com
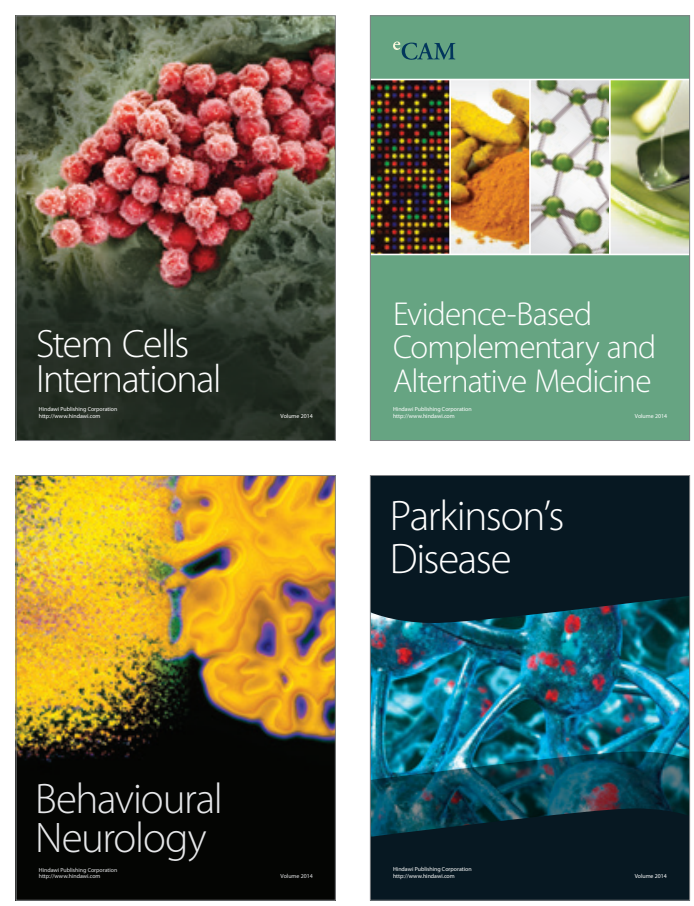

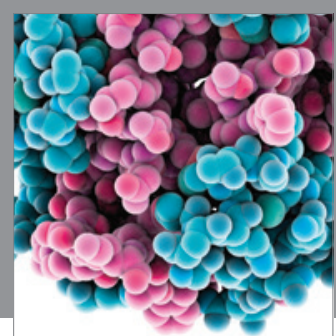

Journal of
Diabetes Research

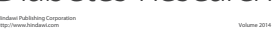

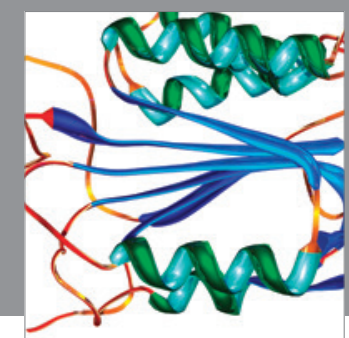

Disease Markers
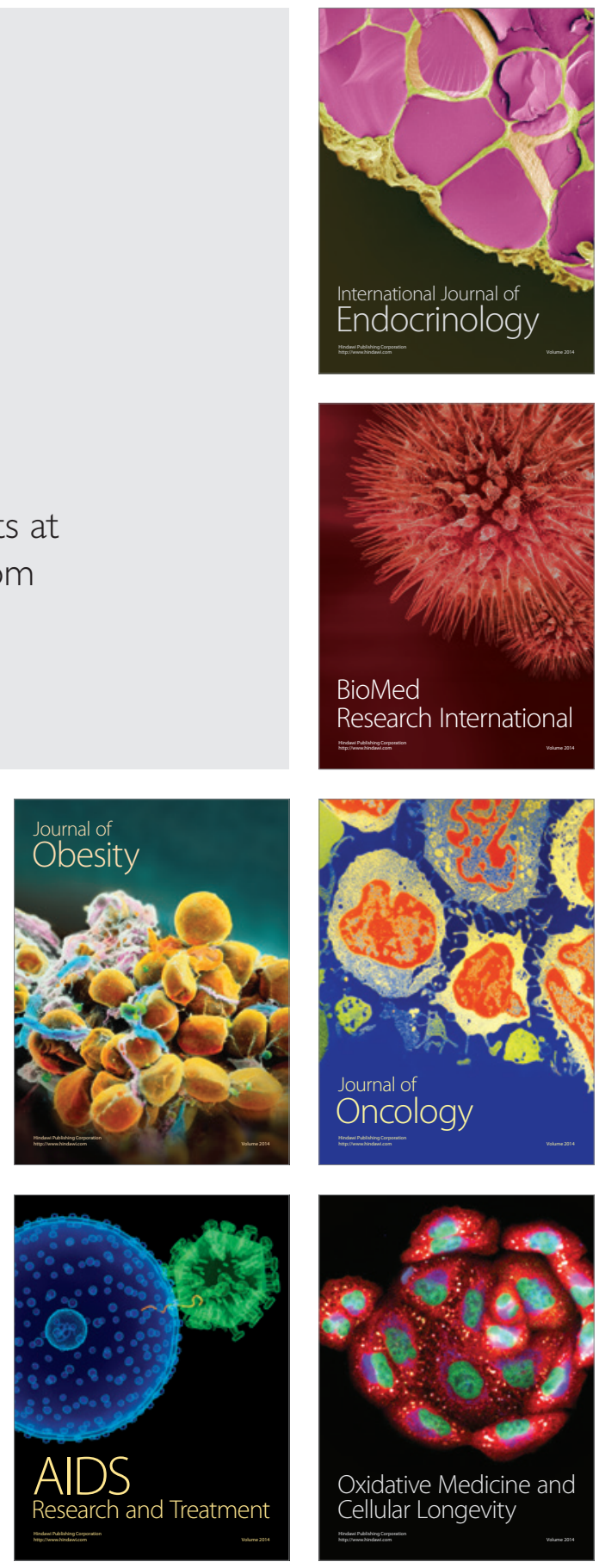